\title{
Probing Disorder in MBE-grown Oxide Films Using Quantitative STEM
}

\author{
Honggyu Kim ${ }^{1}$, Santosh Raghavan ${ }^{1}$, Omor Shoron ${ }^{1}$ and Susanne Stemmer ${ }^{1}$ \\ 1. Materials Department, University of California, Santa Barbara, CA, USA
}

Transparent conducting oxides (TCO) are in great demand for a wide array of optoelectronic devices. Recently, alkaline earth stannates $\left(\mathrm{ASnO}_{3}\right.$, where $\mathrm{A}=\mathrm{Ba}$ or $\left.\mathrm{Sr}\right)$ have emerged as promising candidates for novel TCOs due to their wide optical band gap and high electron mobility. While significant progress has been made in the transport properties of $\mathrm{BaSnO}_{3}$ thin films [1], fewer studies report on $\mathrm{SrSnO}_{3}$ thin films. A major challenge in the investigation on $\mathrm{SrSnO}_{3}$ is the limited conductivity even at relatively high doping, even though chemical doping using La substitution on Sr sites should create a shallow donor. Doping in semiconductors and wide gap oxides can be limited by factors such as low dopant solubility, large ionization energies, charge compensation by point defects, and structural instability associated with dopants and intrinsic point defects. A lack of experimental evidence exists regarding the microscopic origins, which limit doping in such materials. Here we quantify the atomic structure of MBE-grown $\mathrm{SrSnO}_{3}$ using quantitative STEM (QSTEM). Our measurements on atomic column positions reveal structure distortions in the $\mathrm{Sr}$ sub-lattice, but not in the $\mathrm{Sn}-\mathrm{O}$ sub-lattice.

We investigate a La-doped $\mathrm{SrSnO}_{3}$ (space group Pbnm) thin films grown on $\mathrm{PrScO}_{3}$ (space group $\mathrm{Pbnm}$ ) substrate grown by molecular beam epitaxy (MBE) using QSTEM [2], which measures the image intensity on an absolute scale and provides atomic column positions with picometer precision. To minimize image distortion arising from sample drifts and scan noises, a series (20 fast acquisitions) of images were cross-correlated and then averaged. The positions of atomic columns were determined by a 2D Gaussian peak fitting with sub-pixel resolution.

Figure 1(a) shows a representative image of $\mathrm{SrSnO}_{3}$ recorded along the [110]o projection, where the subscript indicates the orthorhombic unit cell. Over the area examined in this work, no extended defects were found. Thus, any nonstoichiometry in the $\mathrm{SrSnO}_{3}$ film is likely to be accommodated by point defects, e.g., vacancies, interstitials, or antisite defects. Intensity maps of the $\mathrm{Sr}$ and $\mathrm{Sn}-\mathrm{O}$ columns [Figs. 1(b,c)] extracted from Fig. 1(a) show gradual intensity variations; there are no distinct signs of point defects, which would result in abrupt intensity changes in the intensity map. Quantitative analysis on displacements of atomic columns was then performed. The angles $\left(\theta_{\mathrm{Sr}}\right.$ and $\left.\theta_{\text {Sn-O }}\right)$ formed by three atomic columns, as depicted in the schematic in Fig. 1(d), serve as a measure of structural distortions. Peak separation method was utilized to obtain $\mathrm{Sr}$ and $\mathrm{Sr}-\mathrm{O}$ sub-lattice images [Figs. 2(a), (c)] from which the $\theta_{\text {Sr }}$ and $\theta_{\text {Sn-O }}$ can be measured [Figs. 2(b), (d)]. In an ideal orthorhombic $\mathrm{SrSnO}_{3}$ along the [110]o projection, the A-site (Sr) displacements are visible as a zig-zag pattern [Fig. 1(d)], resulting in the alternating distribution of the $\theta_{\mathrm{Sr}}$ of $87.14^{\circ}$ and $92.86^{\circ}$, while the $\theta_{\mathrm{Sn}-\mathrm{O}}$ is $90^{\circ}$. The measured $\theta_{\mathrm{Sn}-\mathrm{O}}$ is $89.78 \pm 1.07^{\circ}$, which is close to $89.93 \pm 0.92^{\circ}$ in $\mathrm{SrTiO}_{3}$ (space group $P m \overline{3} m$ ) substrate, a reference. Here, we measure the $\theta_{\mathrm{Sr}}$ of $87.30 \pm 1.84^{\circ}$ and $92.06 \pm 1.60^{\circ}$, i.e., larger deviations from the ideal angles and wider-angle distributions than those of the $\theta_{\text {Sn-O. }}$. Our angle measurement clearly reveals that the lattice distortion dominantly occurs in the Sr sub-lattice. As discussed earlier, either point defects or dopants, or both, are the likely source for lattice distortions in $\mathrm{SrSnO}_{3}$, thus point defects associated with the $\mathrm{Sr}$ sub-lattice, e.g., Sr vacancies, Sn antisite defects and La dopants at Sr sites, can be responsible for lattice distortions and possibly doping limits. To explicitly uncover the origin of point defects, we apply a variable-angle STEM (VA-STEM) [3], which utilizes the angular-dependence of the electron scattering 
and therefore enhance the image contrast and interpretability. The combined approaches of structure and chemical quantifications provide a new opportunity to detect point defects, which cannot be detected by only using either one of those methods [4].

\section{References:}

[1] S. Raghavan et al, APL Materials 4 (2016), 016106.

[2] J. M. LeBeau et al, Phys. Rev. Lett. 100 (2008), 206101.

[3] J. Y. Zhang et al, Sci. Rep. 5 (2015), 12419.

[4] The authors acknowledge support by the U.S. Department of Energy (Grant No. DEFG022ER45994).
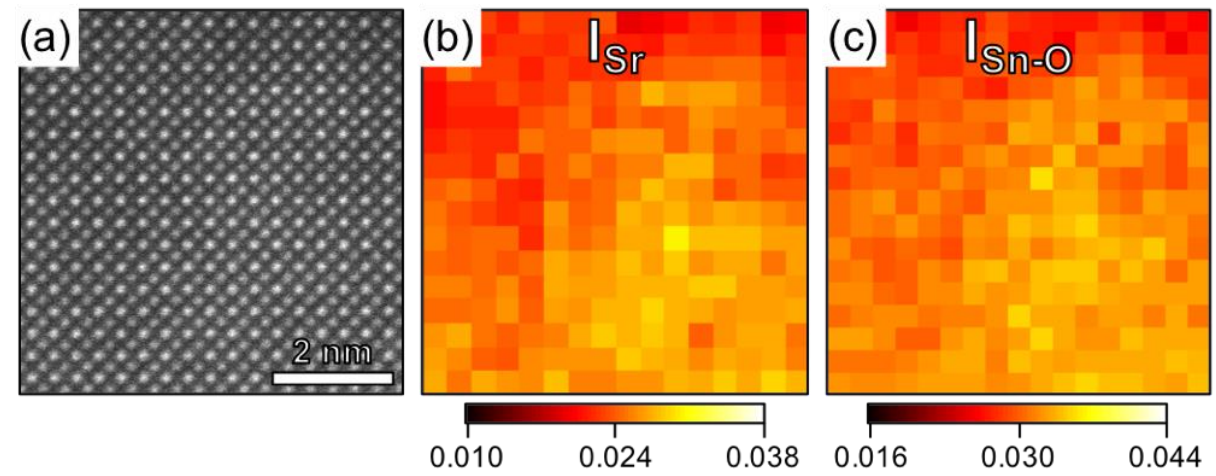

(d)
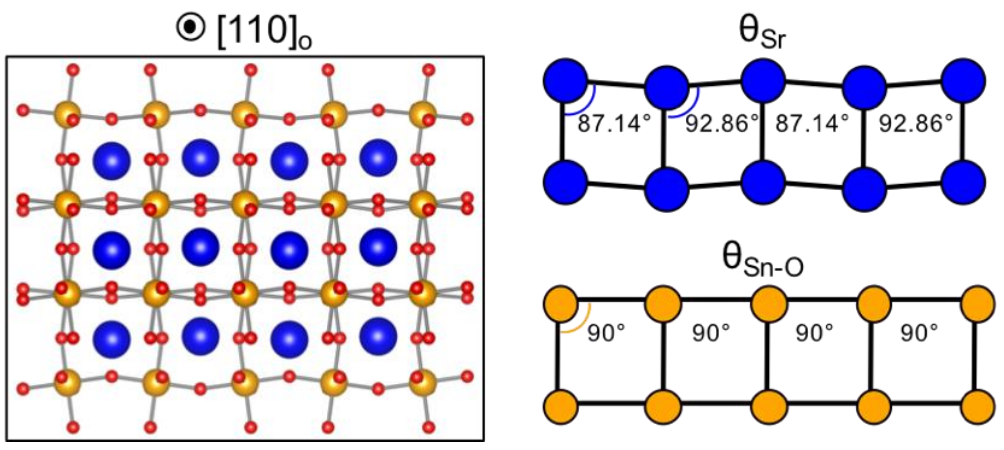

Figure 1. (a) STEM image of $\mathrm{SrSnO}_{3}$. (b) $\mathrm{I}_{\mathrm{Sr}}$ and (c) $\mathrm{I}_{\mathrm{Sn}-\mathrm{O}}$ maps from the image shown in (a). (d) Schematic of $\mathrm{SrSnO}_{3}$ along the [110] projection.

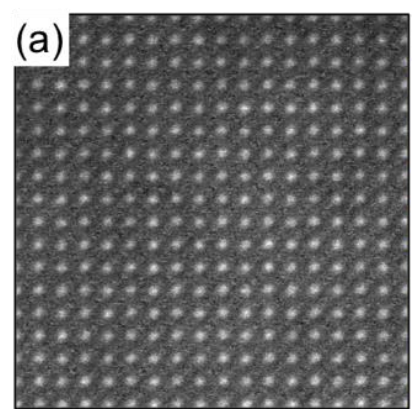

Sr columns

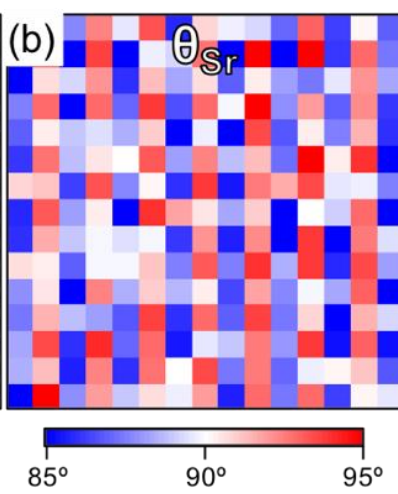

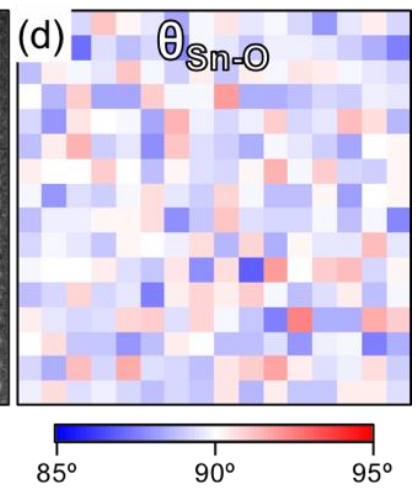

Figure 2. (a,c) STEM images and (b,d) corresponding 2D angle maps of Sr and Sn-O sub-lattices, respectively. 